\title{
Scalable Cryptographic Authentication for High Performance Computing
}

\author{
Andrew Prout, William Arcand, David Bestor, Chansup Byun, Bill Bergeron, Matthew Hubbell, Jeremy Kepner, \\ Andrew McCabe, Peter Michaleas, Julie Mullen, Albert Reuther, and Antonio Rosa \\ MIT Lincoln Laboratory, Lexington, MA 02420 \\ \{aprout, reuther, warcand, cbyun, bbergeron, mhubbell, kepner, amccabe, pmichaleas, jsm, antonio.rosa, \\ david.bestor\}@11.mit.edu
}

\section{INTRODUCTION}

High performance computing (HPC) uses supercomputers and computing clusters to solve large computational problems. Frequently HPC resources are shared systems and access to restricted data sets or resources must be authenticated. These authentication needs can take multiple forms, both internal and external to the HPC cluster. A computational stack that uses web services among nodes in the HPC may need to perform authentication between nodes of the same job or a job may need to reach out to data sources outside the HPC.

Traditional authentication mechanisms such as passwords or digital certificates encounter issues with the distributed and potentially disconnected nature of HPC systems. Distributing and storing plain-text passwords or cryptographic keys among nodes in a HPC system without special protection is a poor security practice. Systems to reach back to the user's terminal for access to the authenticator are possible, but only in fullyinteractive supercomputing where connectivity to the user's terminal can be guaranteed.

Point solutions can be enabled for these use cases, such as software-based role or self-signed certificates, however they require significant expertise in digital certificates to configure. A more general solution is called for that is both secure and easy to use. This paper presents an overview of a solution implemented on the interactive, on-demand LLGrid computing system [3,4,5] at MIT Lincoln Laboratory and its use to solve one such authentication problem.

\section{LINCOLN LABORATORY GRID (LLGRID)}

One of the main design requirements of the LLGrid system was to make HPC usage accessible to the entire Lincoln technical staff by making HPC systems as easy to use as a personal computer. In striving to toward this goal, we developed a system that takes advantage of the desktop development environments (i.e., file system access and integrated development environments like Matlab) with which most engineers and scientists are already familiar. At Lincoln we currently have over 500 users. The vast majority of these users utilize LLGrid in an interactive supercomputing manner. As depicted in Figure 1, interactive supercomputing involves large jobs that require answers in minutes or hours and cannot wait in a queue.

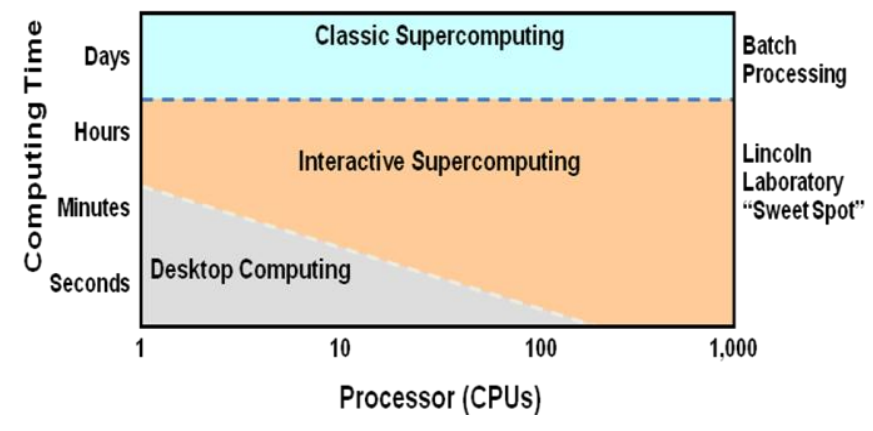

Figure 1: Interactive supercomputing vs. classic supercomputing and desktop computing. Interactive supercomputing jobs require answers in minutes or hours but wait in a queue. Classic supercomputing jobs take hours to days to execute and can tolerate waiting in a queue. Desktop computing jobs take minutes to run on a desktop (e.g. algorithm proof-of-concept).

\section{CRyptographic Key Management On HPC Clusters}

A challenge to any cryptographic key management system is to balance the need to make the keys and their associated Public Key Infrastructure (PKI) certificates available for authorized use, but simultaneously ensure they are properly protected. The most widely used solution for this balance is a smart card that can be accessed using the PKCS\#11 interface standard. While scaling hardware-based smart cards to a HPC environment is not feasible, a cryptographic library that acts similar to a smart card is practical. Several commercial and open source implementations exist, but these focus on different use cases that are tightly coupled with the software packages they ship with, and are not designed to protect keys from export by their authorized user. [1,2]

We developed our own cryptographic library based on the PKCS\#11 standard that connects to a daemon running as a separate user that stores and manages the cryptographic keys for all users on the system. The key storage of this system is designed to allow the keys to only be manipulated by privileged users of the system. Unprivileged users can only access the keys through the PKCS\#11 interface and cannot export them to other locations. This provides security for the cryptographic keys while still allowing them to be used with common security applications such as Transport Layer Security (TLS).
This work is sponsored by the United States Air Force under Air Force contract FA8721-05-C-0002. Opinions, interpretations, conclusions and recommendations are those of the author and are not necessarily endorsed by the United States Government. 
Additionally utilities were developed to manage the keys for users across all nodes in the cluster simultaneously. Key and PKI certificate generation or renewal across hundreds of nodes can be accomplished with a single command in parallel. This allows keys and certificates to be pre-generated for each user so that relying processes can reference them by name on any node they are assigned to run on.

Speed is also an important factor. Commercial smart cards are rated for approximately one operation per second. Reaching back to route cryptographic operations through the user's smart card would quickly bottleneck on this factor. Using a virtual smart card we are only limited by the processing speed of the compute node, capable of over a hundred operations per second per CPU core, and scale linearly.

\section{INTEGRATION WITH SUBVERSION}

This system for cryptographic authentication to external systems was used to enable authenticated access to subversion repositories on the Lincoln Laboratory SourceForge (LLForge) subversion server without putting users' passwords at risk. The subversion client will normally either request to save the user's password in plain text in their home directory or prompt the user to enter their password for each subversion command. Recognizing that widespread storage of passwords in plain text is to be avoided and that frequent prompting of the user to type their password was disruptive, a better solution was sought.

The subversion server's TLS settings for HTTPS were reconfigured to request and PKI certificate authentication and accept the LLGrid PKI certificate authority. The subversion client was configured to attempt authentication using certificates stored in the PKCS\#11 cryptographic library interface discussed above, thus the current LLGrid user identity could be proven to the LLForge subversion server without any user interaction or storage of plaintext passwords.

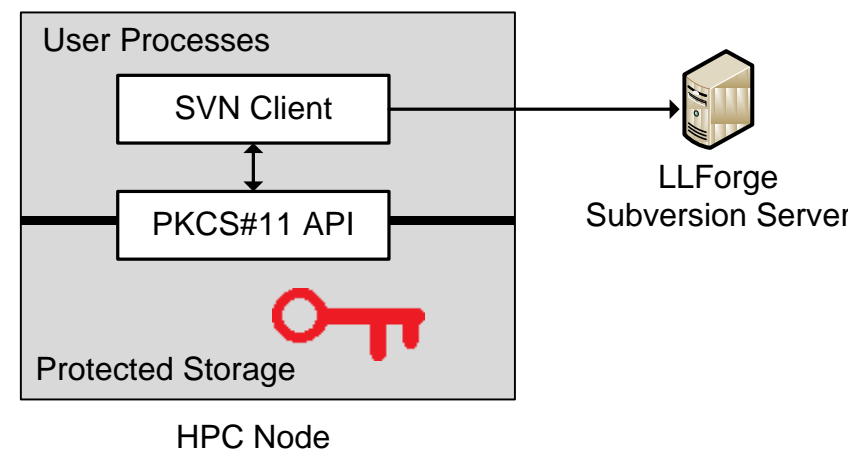

Figure 2: The subversion client running on a HPC node is able to make use of the cryptographic key through the PKCS\#11 interface to prove the user identity to the subversion server, but unable to directly access the protected storage.

\section{Results}

We have built an infrastructure for management of cryptographic key and PKI certificates that can be used for user authentication in HPC environments. This work is applicable to any situation where a scalable method of proving the identity of a HPC process owner to a remote system is needed and can be used to bootstrap trust for other HPC security implementations, such as the work by Foster et al, [6] or to enable the security features of web services as explored by van Engelen. [7] Additionally we have used this system to integrate the LLGrid HPC with the LLForge subversion server to balance ease of use with security.

Future work will investigate the applicability of this solution to other web services operating in HPC or connected to environments.

\section{REFERENCES}

[1] http://live.gnome.org/GnomeKeyring

[2] http://www.mozilla.org/projects/security/pki/nss/

[3] N. Travinin Bliss, R. Bond, J. Kepner, H. Kim, and A. Reuther, "Interactive Grid Computing at Lincoln Laboratory," Lincoln Laboratory Journal, Vol. 16, Number 1, 2006.

[4] A. Reuther, B. Arcand, T. Currie, A. Funk, J. Kepner, M. Hubbell, A. McCabe, P. Michaleas, "TX-2500 - An Interactive, On-Demand RapidPrototyping HPC System,” HPEC 2007, Lexington, MA, Sep. 2009.

[5] A. Reuther, J. Kepner, A. McCabe, J. Mullen, N.T. Bliss, and H. Kim, "Technical Challenges of Supporting Interactive HPC," In Proceedings of the High Performance Computing Modernization Office (HPCMO) Users Group Conference (UGC) 2007, Pittsburgh, PA, 18-22 June 2007.

[6] I. Foster, N. Karonis, C. Kesselman and S. Tuecke, "Managing security in high-performance distributed computations," Cluster Computing, Vol 1, Number 0, 1998.

[7] R. van Engelen, "Pushing the SOAP Envelope With Web Services for Scientific Computing." In proceedings of the International Conference on Web Services (ICWS), pages 346-352, Las Vegas, 2003. 